\title{
PHASE VELOCITY AND ATTENUATION OF LONGITUDINAL SHEAR VIBRATIONS OF HOLLOW POROELASTIC CYLINDERS
}

\author{
S. AHMED SHAH* \\ Department of Mathematics \\ Deccan College of Engineering and Technology \\ Hyderabad - 500001 (A.P), INDIA \\ E-mail: ahmed_shah67@yahoo.com \\ S. JAVVAD HUSSAINI \\ Department of Mathematics \\ Resonance, Kota - 324005 (Rajasthan), INDIA
}

\begin{abstract}
The present paper is devoted to the study of phase velocity and attenuation of longitudinal shear vibrations of hollow poroelastic circular cylinders in the presence of dissipation. The explicit expressions for phase velocity and attenuation of longitudinal shear vibrations are derived. The frequency equation of longitudinal shear vibrations and modes obtained in a previous paper are used to compute the phase velocity and attenuation for different dissipations for thin and thick poroelastic cylindrical shells and poroelastic solid cylinder. The physical parameters of sandstone saturated with kerosene and sandstone saturated with water are used for the purpose of computation. It is found that the phase velocity is linear beyond certain frequency. Phase velocity is smaller for a typical anti-symmetric mode compared to the flexural mode. It is greater for the second mode than that of the first mode. Also the phase velocity is larger for a thin poroelastic cylindrical shell than that of a thick poroelastic cylindrical shell. The same is true for attenuation also. Attenuation is very high for the considered dissipations and it increases with the increase in dissipation.
\end{abstract}

Key words: Biot's theory, hollow cylinder, shear waves, frequency equation, phase velocity, attenuation, wavenumber, dissipation.

\section{Introduction}

In a previous paper (Tajuddin and Shah, 2010), the frequency equation of longitudinal shear vibrations of a hollow poroelastic cylinder of infinite extent was developed that governs the relationship between frequency and the ratio of thickness of the hollow poroelastic cylinder to the inner radius. This frequency equation of longitudinal shear vibrations was obtained in the framework of Biot's (1956) theory of wave propagation in a porous elastic solid under stress free boundary conditions. It was shown that the liquid pressure developed in the solid liquid aggregate is zero and hence the frequency equation of longitudinal shear vibrations is same for pervious and impervious surfaces. This frequency equation was nondimensionalized and frequency was obtained for different values of the ratio of thickness to the inner radius and it was presented in the form of graphs. It was found that these waves are less dispersive. For different values of the radius ratio and different values of angular wavenumber, first five modes were obtained and presented in the form of Tab.1 (Tajuddin and Ahmed Shah, 2010). Also, it was found that the frequency is almost same for Material-I and Material-II each for different values of angular wavenumber, that is, $n=0,1$, 2. Tajuddin and Shah $(2006 ; 2007)$ also studied the circumferential and torsional vibrations of hollow poroelastic cylinders of infinite extent in the presence of dissipation. Shah $(2008 ; 2011)$ studied the problems

\footnotetext{
* To whom correspondence should be addressed
} 
of wave propagation in fluid-filled cylindrical and spherical shells in the absence of dissipation. Recently, Shanker et al. (2013) studied the vibrations of composite poroelastic spheres.

The present paper describes the results of a study prompted by the earlier papers. By using the above said modes (Tab.1, Tajuddin and Shah (2010)), phase velocity and attenuation of longitudinal shear vibrations are obtained for different dissipations and different angular wavenumber for the first two modes for thin poroelastic cylindrical shell, thick shell and poroelastic solid cylinder. The expressions for phase velocity and attenuation are derived involving the dissipation coefficient.

\section{Study of the frequency equation}

A hollow poroelastic circular cylinder of infinite extent is referred to cylindrical polar coordinates. Inner and outer radii of the cylinder are $r_{1}$ and $r_{2}$ respectively so that the thickness of the cylinder is $h\left[=\left(r_{2}-r_{1}\right)>0\right]$. The axis of the cylinder lies along the $z$-axis. The outer and inner surfaces of the hollow cylinder are assumed to be stress free. The frequency equation of longitudinal shear vibrations, independent of the nature of the surface, which was developed in a previous paper (Tajuddin and Ahmed Shah, 2010), is repeated here. This frequency equation is

$$
J_{n}^{\prime}\left(\xi_{3} r_{1}\right) Y_{n}^{\prime}\left(\xi_{3} r_{2}\right)-J_{n}^{\prime}\left(\xi_{3} r_{2}\right) Y_{n}^{\prime}\left(\xi_{3} r_{1}\right)=0
$$

where $J_{n}$ and $Y_{n}$ are Bessel functions of first and second kind, respectively, each of order $n$ and a 'dash' over $J_{n}$ or $Y_{n}$ represents differentiation with respect to $r$. Also $\xi_{3}$ is defined as

$$
\xi_{3}=\frac{\omega}{V_{3}}
$$

In Eq.(2.2), $\omega$ is the circular frequency and $V_{3}$ is the shear wave velocity given by the equation

$$
V_{3}^{2}=\frac{N K_{22}}{K_{11} K_{22}-K_{12}^{2}}
$$

where $N$ is the shear modulus of the poroelastic solid, and

$$
K_{11}=\rho_{11}-\frac{i b}{\omega}, \quad K_{12}=\rho_{12}+\frac{i b}{\omega}, \quad K_{22}=\rho_{22}-\frac{i b}{\omega} .
$$

In Eq.(2.4), $b$ is dissipation coefficient, $\rho_{i j}(i, j=1,2)$ are mass coefficients following Biot (1956) and $i$ is complex unity or $i^{2}=-1$.

By substituting

$$
R_{m}=\xi_{3} h
$$

in Eq.(2.1), it is reduced to

$$
J_{n}^{\prime}\left[\frac{R_{m}}{(g-l)}\right] Y_{n}^{\prime}\left[\frac{R_{m} g}{(g-l)}\right]-J_{n}^{\prime}\left[\frac{R_{m} g}{(g-l)}\right] Y_{n}^{\prime}\left[\frac{R_{m}}{(g-l)}\right]=0
$$


where the roots of Eq.(2.6) are $R_{m}$ which is the $m$-th mode for different values of $g=r_{2} / r_{l}$ and different values of $n$. These values of $R_{m}$ are given in Tab.1 of the previous paper (Tajuddin and Ahmed Shah, 2010).

Using Eqs (2.2)-(2.3) in Eq.(2.5), it is simplified to

$$
R_{m}^{2}=\frac{\omega^{2} h^{2}\left(K_{11} K_{22}-K_{12}^{2}\right)}{N K_{22}}
$$

Now we introduce the following non-dimensional parameters for further simplification

$$
m_{11}=\frac{\rho_{11}}{\rho}, \quad m_{12}=\frac{\rho_{12}}{\rho}, \quad m_{22}=\frac{\rho_{22}}{\rho}, \quad b_{1}=\frac{b h}{\rho C_{0}}, \quad \Omega=\frac{\omega h}{C_{0}}
$$

where $b_{1}, \Omega$ are non-dimensional dissipation, frequency respectively, $C_{0}^{2}=\frac{N}{\rho}$ is the reference velocity and $\rho=\rho_{11}+2 \rho_{12}+\rho_{22}$.

Equation (2.7) is written as

$$
\frac{R_{m}^{2} N}{\omega^{2} h^{2}}=\frac{\left(K_{11} K_{22}-K_{12}^{2}\right)}{K_{22}}
$$

where $K_{11}, K_{12}$ and $K_{22}$ are defined in Eqs (2.4).

But it is seen that $\frac{\left(K_{11} K_{22}-K_{12}^{2}\right)}{K_{22}}=\rho\left[E_{r}-i E_{i}\right]$, and thus we get

$$
\frac{R_{m}^{2} N}{\omega^{2} h^{2}}=\rho\left[E_{r}-i E_{i}\right]
$$

where $E_{r}$ and $E_{i}$ are

$$
E_{r}=\frac{\Omega^{2} m_{22}\left(m_{11} m_{22}-m_{12}^{2}\right)+b_{1}^{2}}{\Omega^{2} m_{22}^{2}+b_{1}^{2}}, \quad E_{i}=\frac{\Omega b_{1}\left(m_{12}+m_{22}\right)^{2}}{\Omega^{2} m_{22}^{2}+b_{1}^{2}} .
$$

A poroelastic medium is dissipative in nature and thus the axial wavenumber $k$ is complex. The waves generated obey the diffusion type process and therefore get attenuated. Let $n=k h$ and $k=k_{r}+i k_{i}$, where $k_{r}$ is real and $k_{i}$ is the imaginary part of the wavenumber $k$. Hence the phase velocity $C_{p}$ and attenuation $x_{h}$ as in Biot (1956) are

$$
C_{p}=\frac{\omega}{\left|k_{r}\right|}, \quad x_{h}=\frac{1}{\left|k_{i}\right|}
$$

By substituting $n=k h$ in Eq.(2.10) it reduces to 


$$
\frac{R_{m}^{2} N k^{2}}{\rho \omega^{2} n^{2}}=\left[E_{r}-i E_{i}\right]
$$

Now by substituting $k=k_{r}+i k_{i}$, in Eq.(2.13) and after necessary simplifications, we can separate the real and imaginary parts of the wavenumber as

$$
k_{r}=\frac{1}{\sqrt{2} h}\left(B_{1}+B_{2}\right)^{\frac{1}{2}}, \quad k_{i}=\frac{1}{\sqrt{2} h}\left(B_{1}-B_{2}\right)^{\frac{1}{2}}
$$

where the expressions for $B_{1}$ and $B_{2}$ are

$$
B_{1}=\frac{\Omega^{2} n^{2}\left(E_{r}^{2}+E_{i}^{2}\right)^{\frac{1}{2}}}{R_{m}^{2}}, \quad B_{2}=\frac{\Omega^{2} n^{2} E_{r}}{R_{m}^{2}} .
$$

In Eq.(2.15), $E_{r}$ and $E_{i}$ are defined in Eq.(2.11).

Thus the non-dimensional phase velocity $\frac{C_{p}}{C_{0}}$ and attenuation $\frac{x_{h}}{h}$ are

$$
\frac{C_{p}}{C_{0}}=\sqrt{2} \Omega\left(B_{1}+B_{2}\right)^{-\frac{1}{2}}, \quad \frac{x_{h}}{h}=\sqrt{2}\left(B_{1}-B_{2}\right)^{-\frac{1}{2}}
$$

where $B_{1}$ and $B_{2}$ are defined in Eq.(2.15).

\section{Results and discussion}

Two types of poroelastic materials are considered to carry out the computational work, one is sandstone saturated with kerosene, say Material-I (Fatt, 1959), the other one is sandstone saturated with water, say Material-II (Yew and Jogi, 1976), whose non-dimensional physical parameters are given in Tab.1

Table 1.

\begin{tabular}{|l|c|c|c|c|c|}
\hline Material/parameter & $a_{4}$ & $\tilde{z}$ & $m_{11}$ & $m_{12}$ & $m_{22}$ \\
\hline Material-I & 0.234 & 3.851 & 0.901 & -0.001 & 0.101 \\
\hline Material-II & 0.412 & 2.129 & 0.877 & 0 & 0.123 \\
\hline
\end{tabular}

For a given poroelastic material, the non-dimensional phase velocity and attenuation (damping coefficient) are computed as a function of frequency for the first two modes. The different dissipation parameters $\left(b_{1}\right)$ chosen are $0.01,0.1$ and 1 . Three values of $g$ have been considered for the purpose of computation. These values are $g=1.034,3$ and when $g$ is very large (i.e., $g \rightarrow \infty$ ). These three cases physically represent the results related to the poroelastic thin shell, thick shell, poroelastic solid cylinder, respectively. Different angular numbers considered for computation are $n=1$ and $n=2$ which respectively represent the flexural mode and typical anti-symmetric mode. Phase velocity of a thin poroelastic circular cylindrical shell Material-I is presented in Fig.1 for the considered dissipations, angular wave numbers and the first two modes. It is seen from Fig. 1 that the phase velocity increases till the frequency is 0.1 and then it remains almost constant. In general, phase velocity is high for lower dissipation. Phase velocity is high for second mode that that of the first mode. Also, phase velocity for $n=1$ is higher than that of $n=2$. Phase velocity of a thin poroelastic circular cylindrical shell in case of Material-II is presented in Fig.7. The 
variation of phase velocity is similar as discussed in Fig.1. Also, phase velocity is almost same for both the materials. Thus it is clear that the mass-coupling parameter has no significant effect on phase velocity in the case of a thin poroelastic cylindrical shell. Attenuation of a thin poroelastic cylindrical shell for $n=1$ and 2 is presented in Fig.2 for Material-I for the first mode. It is seen that as dissipation increases, attenuation increases. There is an increase in attenuation when the frequency is in $\left[\begin{array}{ll}0 & 0.1\end{array}\right]$ for all dissipations and then there is a rapid decrease. Attenuation for $n=1$ is higher than that for $n=2$. The same trend is observed in variation of attenuation of the thin poroelastic cylindrical shell for the second mode for Material-I. Moreover, attenuation is higher for second mode than that of the first mode for all the considered dissipations. The phase velocity of a thick poroelastic cylindrical shell for Material-I is presented in Fig.3 for the considered dissipations and for $n=1,2$. The variation of phase velocity is similar as that discussed in Fig.1. It is observed that the phase velocity for a thick shell is less than that for a thin shell. The phase velocity of a poroelastic solid cylinder for Material-I is presented in Fig. 5 for the considered dissipations and for $n=1,2$. Variation of phase velocity is similar to that discussed in Fig.1. Here we can say that the increase in thickness of the hollow poroelastic cylindrical shell has no effect on phase velocity since phase velocity is high for a thin shell and it is smallest for a thick shell while the phase velocity of a solid cylinder is higher than that of the phase velocity of a thick shell. Exactly the same trend is observed in variation of phase velocity in case of Material-II and moreover the phase velocity for Material-II is almost same as that of Material-I. Attenuation of a thick poroelastic shell is presented in Fig. 4 for Material-I for $n=1,2$ and for the first mode. Variation of attenuation is similar as discussed in Fig. 2 and it is higher for a thin shell than that for a thick shell. Similarly, attenuation of a poroelastic solid cylinder for Material-I and for the first mode with considered dissipations is presented in Fig.6. Again the variation of attenuation is same as that discussed in Fig.2. Attenuation of a solid cylinder is larger than that of attenuation of a thick shell but it is smaller than that of attenuation of a thin poroelastic cylindrical shell. Attenuation for the second mode is higher than that of the first mode. Same trend is observed in variation of attenuation in case of Material-II. Thus the mass-coupling parameter has no significant effect on attenuation.

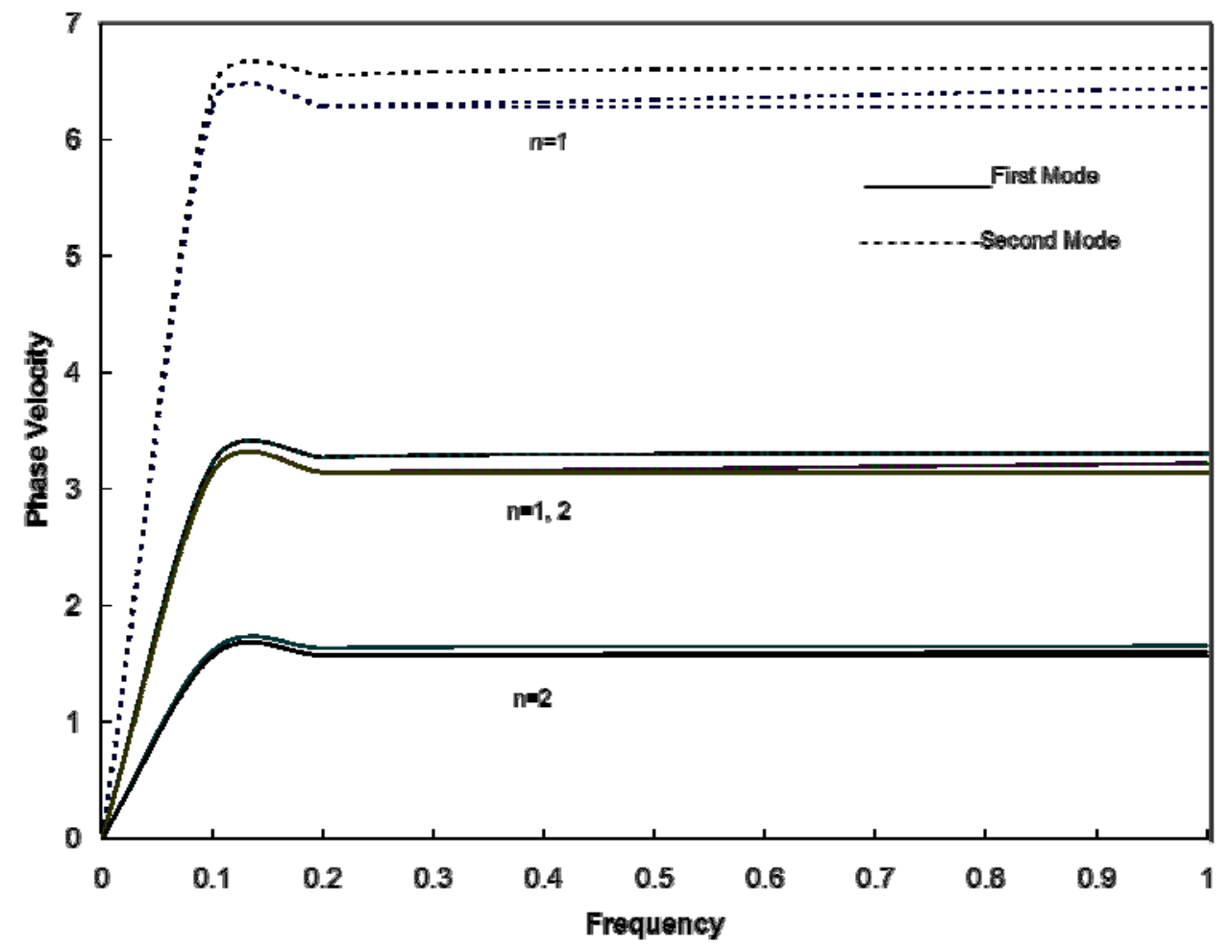

Fig.1. Phase velocity as a function of frequency (Mat-I, Thin-shell). Longitudinal shear vibrations of hollow poroelastic cylinders. 


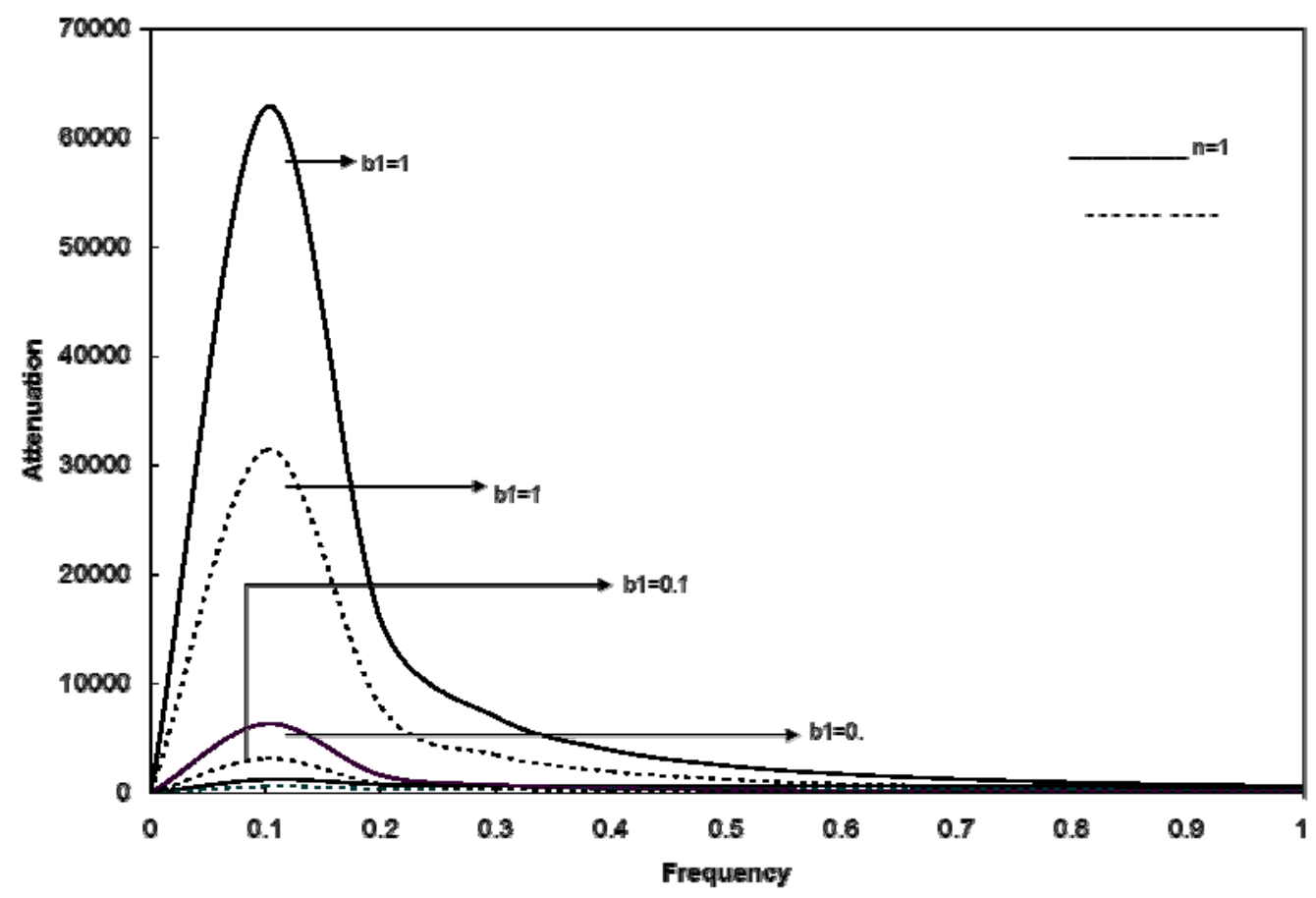

Fig.2. Attenuation as a function of frequency (Mat-I, Thin-shell, First mode). Longitudinal shear vibrations of hollow poroelastic cylinders.

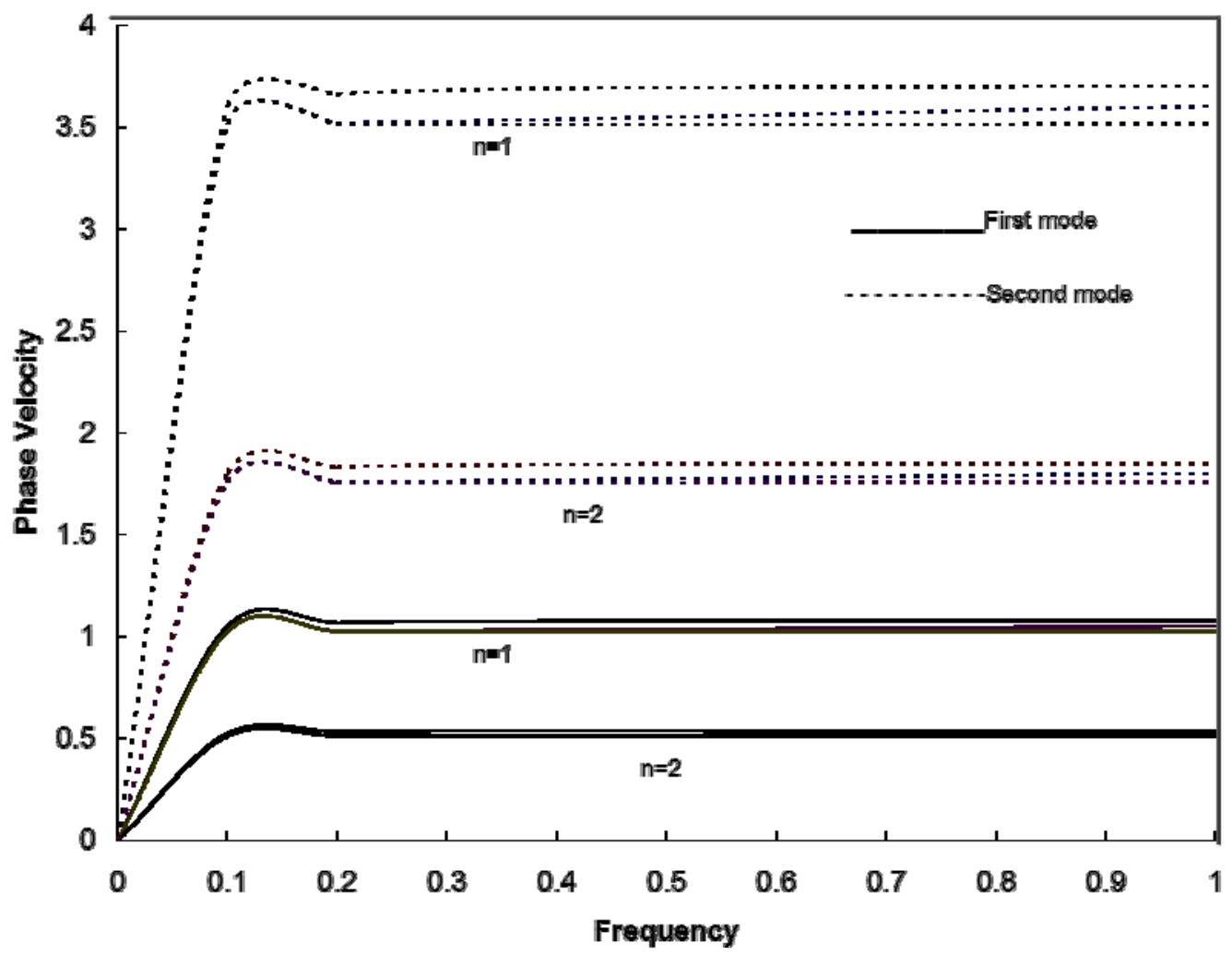

Fig.3. Phase velocity as a function of frequency (Mat-I, Thin-shell). Longitudinal Shear vibrations of hollow poroelastic cylinders. 


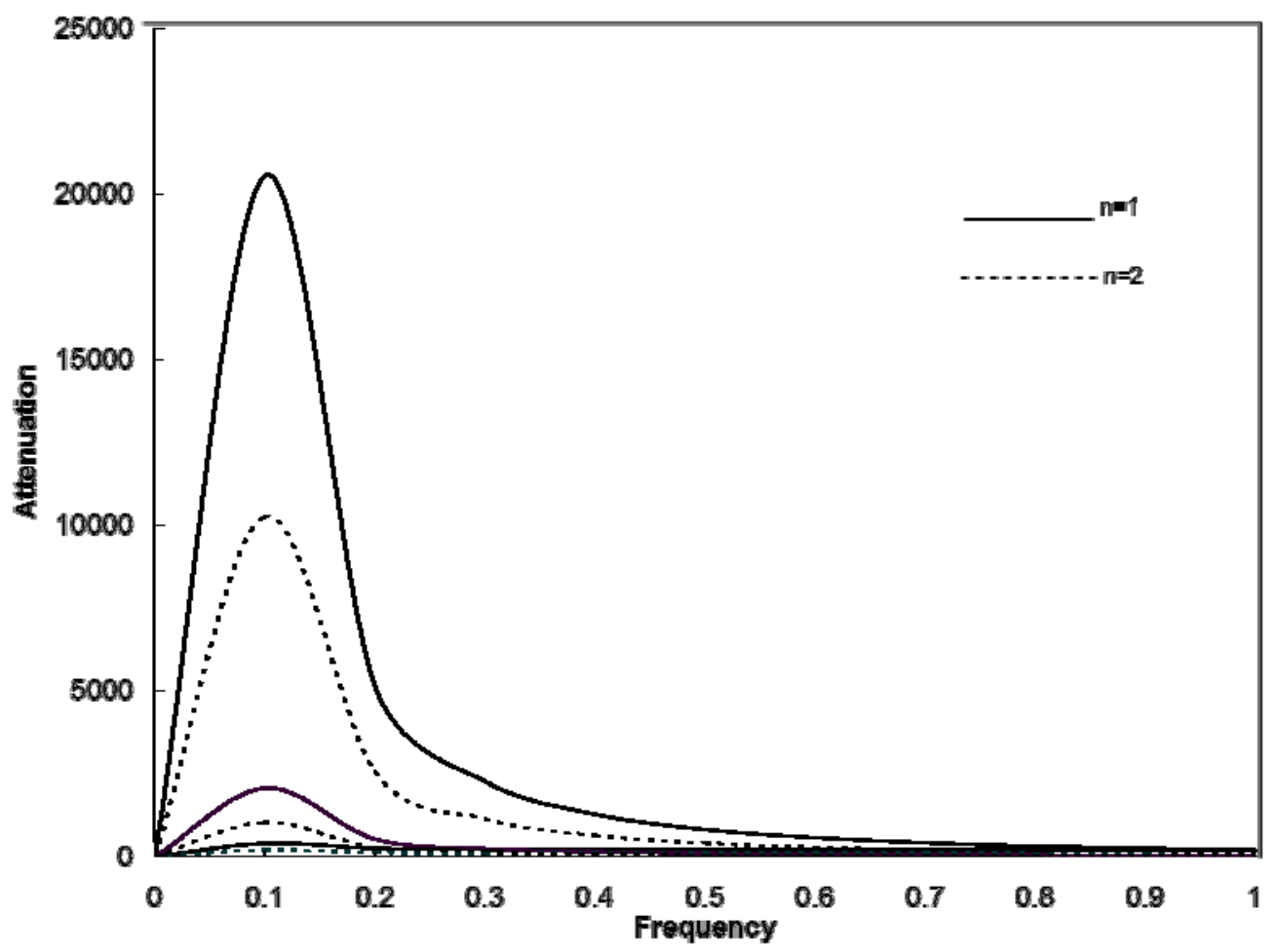

Fig.4. Attenuation as a function of frequency (Mat-I, Thin-shell, First mode). Longitudinal shear vibrations of hollow poroelastic cylinders.

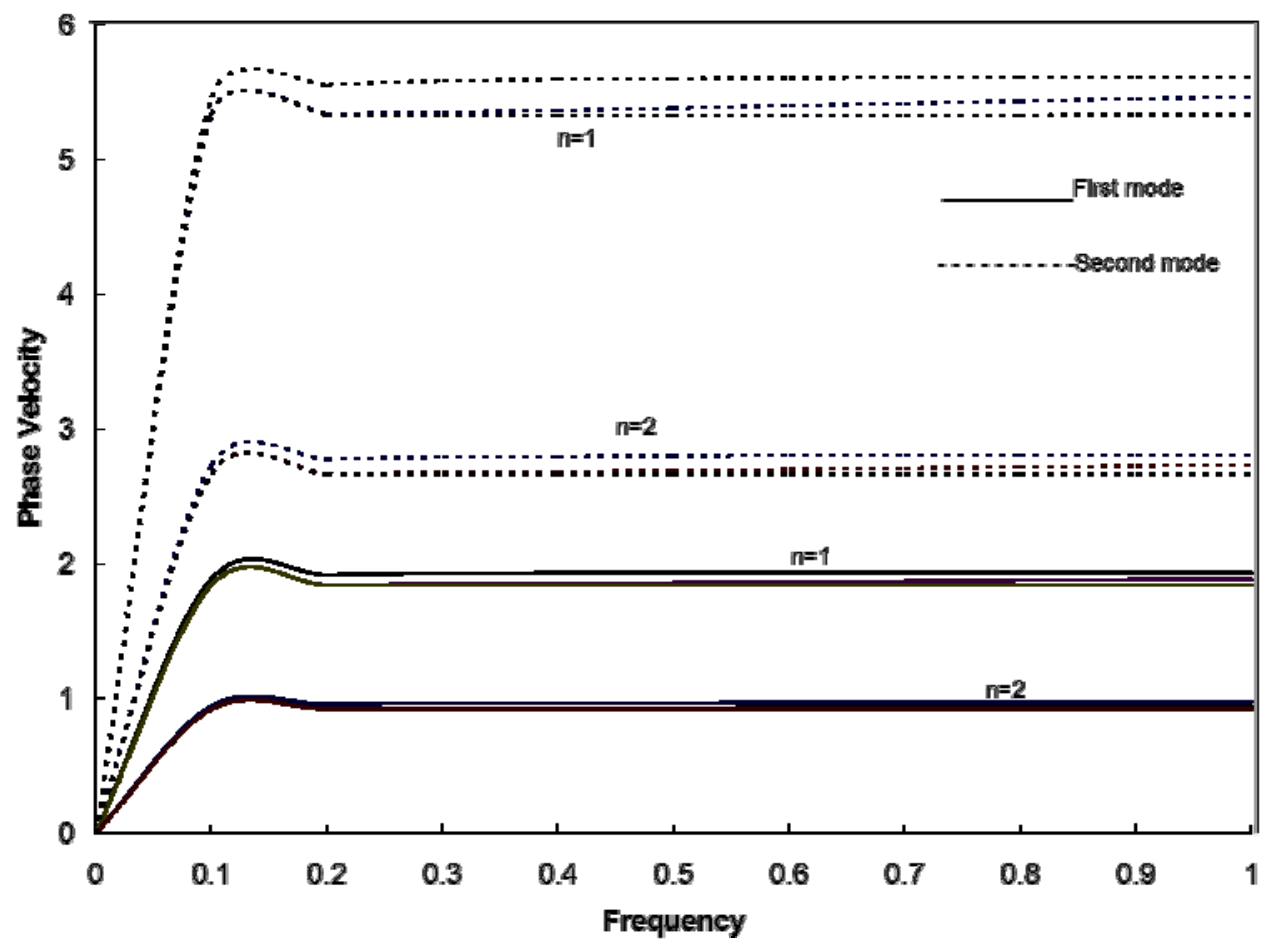

Fig.5. Phase velocity as a function of frequency (Mat-I, Solid cylinder). Longitudinal shear vibrations of hollow poroelastic cylinder. 


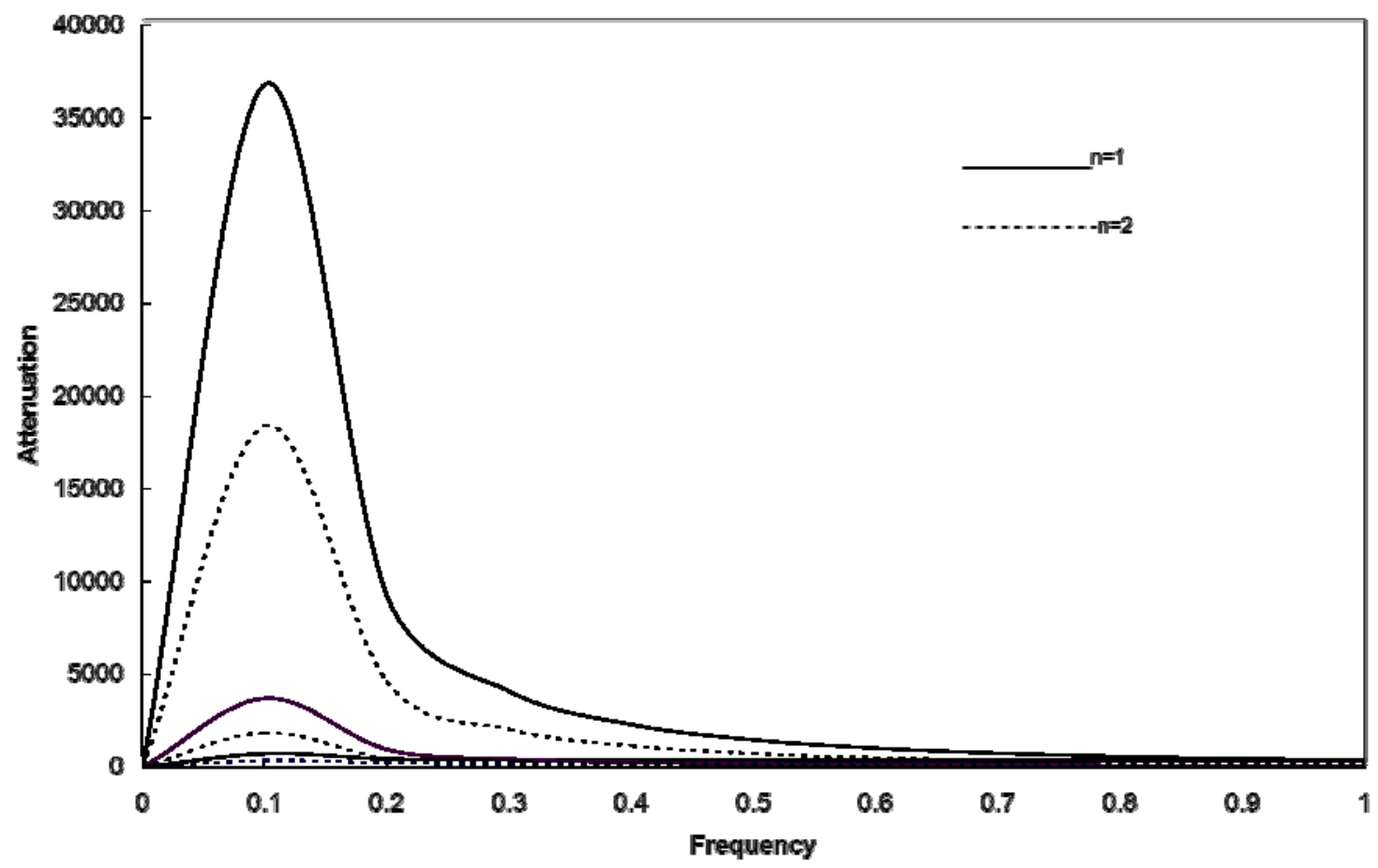

Fig.6. Attenuation as a function of frequency (Mat-I, Solid cylinder, First mode). Longitudinal shear vibrations of hollow poroelastic cylinders.

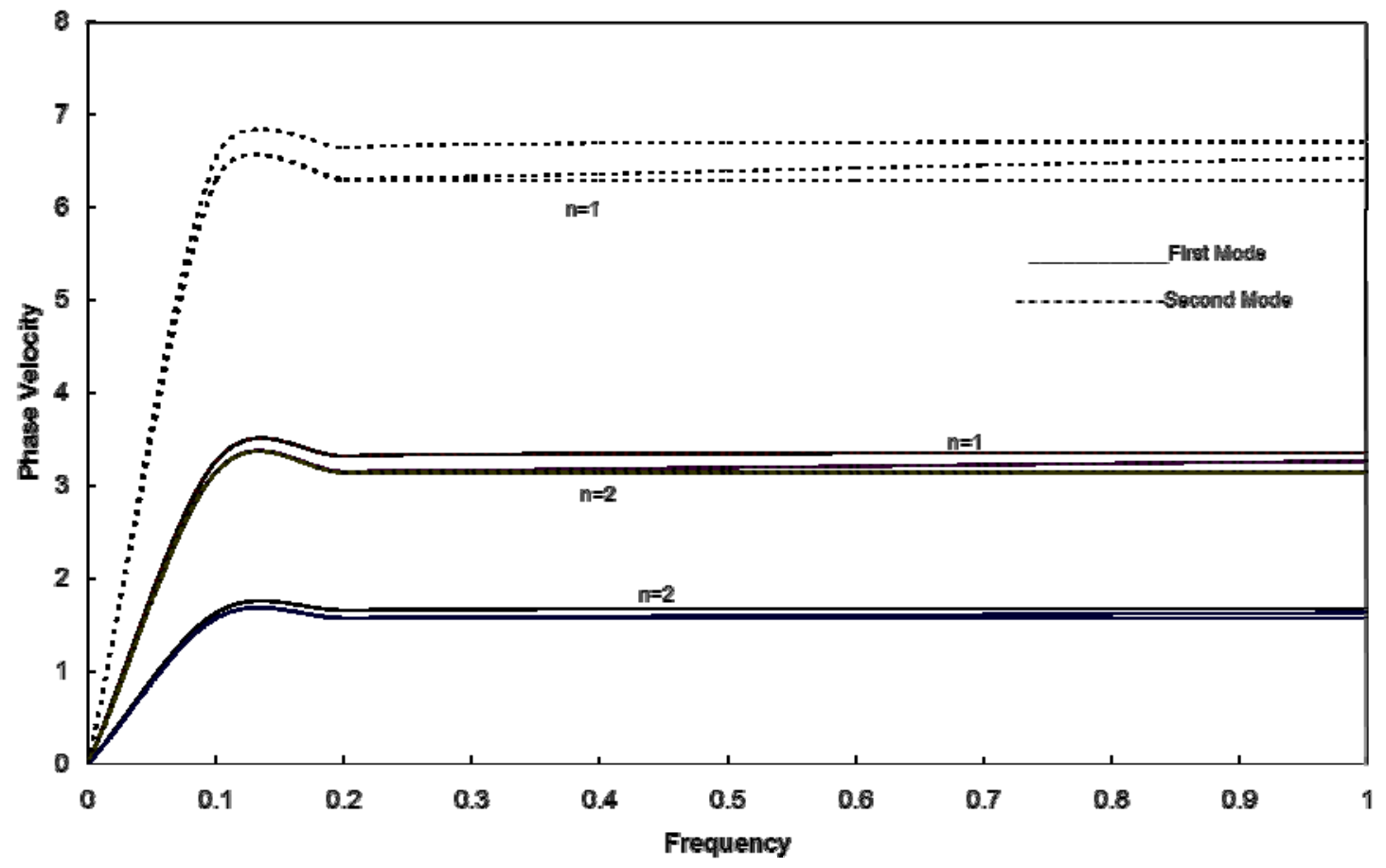

Fig.7. Phase velocity as a function of frequency (Mat-II, Thin-shell). Longitudinal shear vibrations of hollow poroelastic cylinders. 


\section{Concluding remarks}

(i) Phase velocity and attenuation for flexural mode is higher than that of typical anti symmetric mode.

(ii) With the increase in dissipation, there is no change in phase velocity

(iii) With the increase in dissipation, there is an increase in attenuation.

(iv) Phase velocity and attenuation is higher for the second mode that that of the first mode.

(v) The phase velocity and attenuation is highest for a thin poroelastic cylindrical shell and these are lowest for a thick poroelastic shell.

(vi) Mass-coupling parameter has no significant effect on phase velocity and attenuation.

\section{Nomenclature}

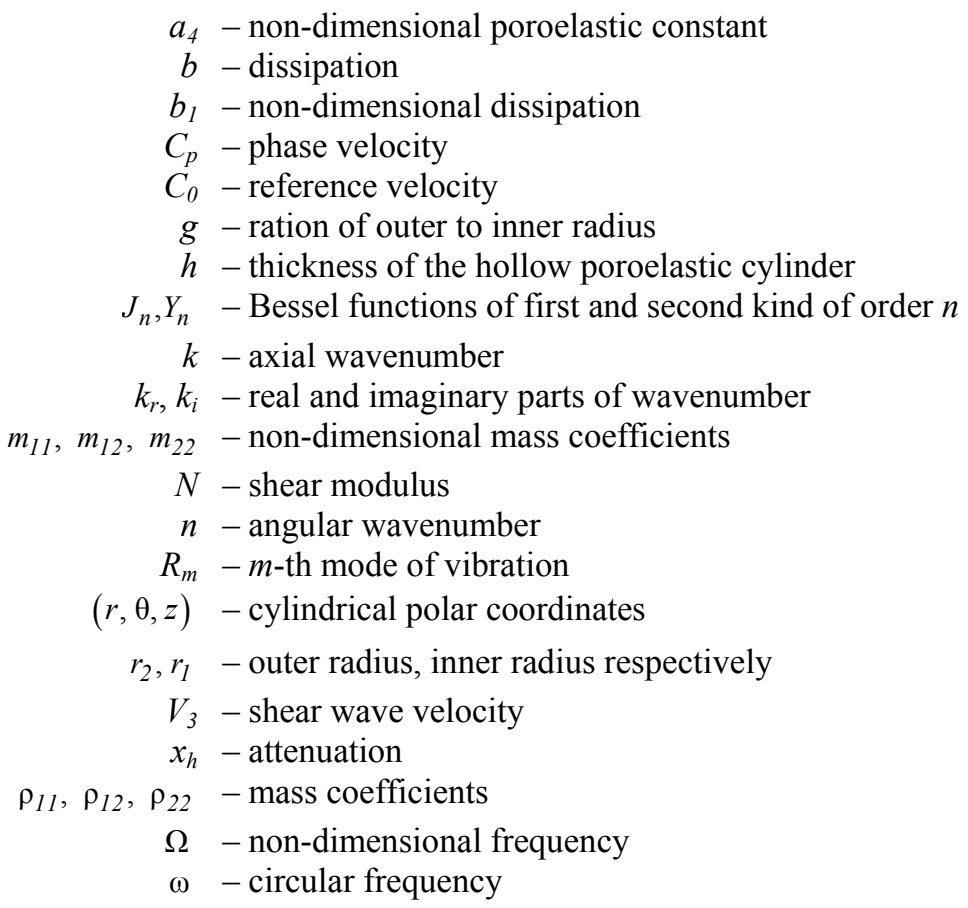

\section{References}

Ahmed Shah S. (2008): Axially symmetric vibrations of fluid-filled poroelastic circular cylindrical shells. - Journal of Sound and Vibration, vol.318, pp.389-405.

Ahmed Shah S. and Tajuddin, M. (2011): On axially symmetric vibrations of fluid filled poroelastic pherical shells. Open Journal of Acoustics. vol.1, pp.15-26.

Biot M.A. (1956): Theory of propagation of elastic waves in fluid-saturated porous solid. - J. Acoust. Soc. Am., vol.28, pp.168-178.

Fatt I. (1959): The Biot-Willis elastic coefficients for a sandstone. - J. Appl. Mech., vol.26, pp.296-297.

Shanker B., Nageswara Nath C., Ahmed Shah S. and Manoj Kumar J. (2013): Vibration analysis of a poroelastic composite hollow sphere. - Acta Mechanica, vol.224, pp.327-341.

Tajuddin M. and Ahmed Shah S. (2006): Circumferential waves of infinite hollow poroelastic cylinders. - Trans. ASME, J. Appl. Mech., vol.73, pp.705-708. 
Tajuddin M. and Ahmed Shah S. (2007): On torsional vibrations of infinite hollow poroelastic cylinders. - Journal of Mechanics of Materials and Structures, vol.2, pp.189-200.

Tajuddin M. and Ahmed Shah S. (2010): Longitudinal shear vibrations of hollow poroelastic cylinders. - Bulletin of Cal. Math. Soc., vol.102, pp.289-298.

Yew C.H. and Jogi P.N. (1976): Study of wave motions in fluid-saturated porous rocks. - J. Acoust. Soc. Am., vol.60, pp.2-8.

Received: August 23, 2013

Revised: October 14, 2013 